\title{
A Mobile Platform For Food Donation AND DELIVERY SYSTEM USING AI AND MACHINE LEARNING
}

\author{
George Zhou ${ }^{1}$, Marisabel Chang ${ }^{2}$ and Yu Sun ${ }^{2}$ \\ ${ }^{1}$ Santa Margarita Catholic High school, \\ 22062 Antonio PkwyRancho Santa Margarita, CA 92688 \\ ${ }^{2}$ California State Polytechnic University, Pomona, CA, 91768
}

\begin{abstract}
Within the last year through the turmoil of the Covid-19 pandemic, an increasing number of families and individuals are experiencing food insecurity due to a loss of job, illnesses, or other financial struggles [4]. Many families in the Orange County area and abroad are turning to free food sources such as community food pantries or banks. Using specified surveys to food insecure families, we discovered a need for a solution to enhance the accessibility and usability of food pantries [5]. Therefore, we created a software application that uses artificial intelligence to locate specific items for users to request, and allow volunteers to see those requests and pick up the resources from food pantries, and deliver them directly to the homes of individuals. This paper shows the process in which this idea was created and how it was applied, along with the conduction of the qualitative evaluation of the approach. The results show that the software application allowed families and individuals to receive quality groceries at a much higher frequency, regardless of multiple constraints.
\end{abstract}

\section{KEYWORDS}

Mobile Platform, machine learning, data mining.

\section{INTRODUCTION}

A growing number of people are out of employment [6]. Hunger and food insecurity has grown dramatically due to healthcare and the economic crisis caused by the Corona virus [7]. Before the pandemic, more than 35 million Americans lived in households that struggled against hunger, and one in ten (10.5 percent) of households in the U.S. will experience food insecurity in 2019. Due to the effects of the corona virus pandemic, this number has grown to more than 42 million. People who may experience food insecurity, including a potential 13 million children according to USDA and Feeding America, may reside in surprisingly wealthy communities or demographics, but because of the recent pandemic, have recently been in financial strules. In Orange County, the average household experiencing food insecurity grew from $8.9 \%$ to $12.1 \%$ in 2019 to 2020, a total of 427,058 households. Many households that experience food insecurity do not qualify for federal nutrition programs and need to rely on their local food banks and other hunger relief organizations for support [14]. Therefore, there has been an increased need for free resources not coming directly from the government, such as food pantries, food bands, food drives, and or local distributions. Food pantries across the nation, specifically in Orange county, have seen drastic increases in visits and food distributed. Taking our Crossline Church food pantry as an example, we distributed 2.1 million $\mathrm{lbs}$ in 2021, growing from $\$ 125,000 \mathrm{lbs}$ in 2019, that is $1,600 \%$ growth. In the first quarter of 2021, we've already outpaced the first quarter of

David C. Wyld et al. (Eds): COMIT, CRBL, BIOM, WiMNeT, SIP, AISO - 2021 
2020, distributing 586,754 lbs of food in Q1 of 2021 versus 95,510 lbs from Q1 of 2020, another $500 \%$ growth.

There is no direct competition that exactly matches our business model. However there are few related products and services that are inline with our mission, including Food Bank's mobile food pantries are set up at sites that are close to the communities that have a higher concentration of vulnerable families [8].However it differs from our business model in that the mobile food pantries are still limited and can not cover an unlimited geographical area like our application can. Additionally, the mobile food pantries do not have a digital platform that connects to the Clients directly [9]. Therefore, the supply and demand is not connected and therefore has the same limitation like traditional food pantries [10]. Another application that has similar methodologies include the MealConnect App, which connects food banks with restaurants and grocery stores. The app matches the excess food at the restaurants and grocery stores with local food banks to reduce food waste. However the App does not connect directly with Clients, nor deliver to them directly. Delivery with Dignity Orange County program connects the restaurants with the volunteers through non-profit agencies to help deliver food to people who need the program based on "Triple Threat" criteria. This includes those that are at the highest risk for COVID-19 per CDC guidelines, as well as those who are ineligible or have not served by any community organization for the provision of food to their homes. It also includes families or individuals that are financially unable to meet their food needs without leaving their home, and who do not have a reliable support system of friends or family to assist. You could see that this effort does not have the digital platform and resources like that of our application, and it also does not connect directly with those individuals and catering to their needs.

PantryGo is a data driven, easy to use, smart delivery app that responds to general food insecurity as well as the healthcare and economic crisis created by the novel coronavirus. It works to assist our "Clients", the most vulnerable and isolated individuals in OC who are not able to reach quality food sources due to various constraints through partnerships with "Services providers", including local food pantries, grocery stores, and leading nonprofit agencies [11]. The program recruits "Volunteers" to deliver meals, pantry food, and excess groceries directly to the doorsteps of Clients. Simultaneous to feeding those in need, PantryGo is reducing the food waste in food pantries and grocery stores by providing estimated demand, food shelf time, food availability, resources allocation and other Artificial Intelligent information through the app. The strengths of PantryGo compared to its competitors include its reliability, which means that clients can count on PantryGo to quickly deliver fresh food and do not have to question whether proper food supply is available. Service Providers can count on PantryGo to know what and how much supplies are needed to meet the needs of those families. Next is its operational efficiency, meaning that PantryGo is the fastest service or delivery among food pantries. Technology is not widely used in the food pantry realm, or any service providing free resources to the most vulnerable. The uniqueness of this product will help food pantries, grocery stores, and all Service Providers to serve their Clients better, by saving their time, saving gas, saving resources, through one platform. Another strength is the quality of PantryGo, in which it serves all types of people in any circumstance. Regardless of physical, mental, or geographical constraints, the app allows any person to receive quality food and nutrition. Instead of quick meals from fast food restaurants or cheap, unhealthy snacks, the app allows for simple, quick, free deliveries of fresh produce. Additionally, the data that PantryGo provides is a SnapShot of hunger and food insecurity in any given community, that helps identify the numerous informational data regarding the behavior of the clients. This includes the most frequently requested items, as well as a spread chart on the locations of these clients and average delivery time for volunteers. This information can be used by food pantries and other agencies to better understand the tendencies and behavior of these families, so they can mold their programs or services to these findings. 
Lastly, PantryGo allows for a stronger community, in which it helps our churches, government agencies, and policy makers to allocate the resources and tackle the root cause of food insecurity. Management teams can oversee, perform and solve problems better than before.

To prove our results, we can look at the percentage of which food waste has been reduced within the food bank system and individual food pantries, as well as the number of food received by food insecure families and individuals. In finding the decrease in the amount of food waste within the food bank system and individual food pantries, we can compare the current amount of food waste left at food banks, pantries, restaurants, and grocery stores compared to the amount of food waste left over after the implementation of the app. To find the percentage decrease of the food waste, we can divide the final amount by the original amount. This percentage will show the effectiveness of our application, furthermore proving our results in finding a useful solution. PantryGo, as stated before, hopes to greatly reduce food waste and surplus amounts of food that go to waste, as it is negatively impacting the environment, while simultaneously out of reach for families and individuals who desperately need those resources. Therefore, in measurement of the decrease in food waste that was able to reach these families and individuals, we can determine the success of our solution and furthermore prove our results. Additionally, in finding the number of food insecure families and individuals who are receiving more food after the launch of PantryGo, we can distribute online surveys to question whether their food supply has increased or decreased [12]. Because of the easy to use nature of the application, and the convenience in which it offers, families will be able to receive food at a much higher rate.

The rest of the paper is organized as follows: Section 2 explains the details and description of the challenges that were faced during the experiment, as well as the development and design of the solution. Next is section 3, which discusses the solution that was developed in wake of the topic and the methodology of that solution. Following that is section 4, which focuses on the experiment itself and an evaluation of that experiment in its process, followed by section 5 , which discusses related works within the same field or topic that will be presented and evaluated. Finally, section 6 presents the concluding remarks and ideas, as well as future work intended for this project.

\section{Challenges}

In order to build the tracking system, a few challenges have been identified as follows.

\subsection{Finding a Solution}

One of the first and biggest challenges that we faced in solving the problems that re-occurred at the food pantry was finding the solution itself. The food bank system along with the idea of a food pantry have existed for a few decades already, and since its creation, no one has come up with a solution to improve the reach of the food pantry and bring it to a technological level. Therefore, in the early stages of PantryGo, it was difficult finding the right structure and system for the application and the process in which the food would be delivered. Furthermore, in finding a solution, we did not want to alter the system on which the food pantry is already operating, as many volunteers have spent countless hours and days perfecting the system in place. After many weeks of brainstorming and adjustment, we were able to keep the current food bank system while moving the system to a digital platform and expanding the reach and accessibility of the food pantry system. 


\subsection{Creating the App}

Most likely the biggest challenge of the experiment/project is the actual designing, development, and engineering of the mobile application within PantryGo. As a coder who only had a few weeks of experiment under the belt, developing a professional application was extremely hard from the get go. The implementation of code and constant refinement of the design as well as the UI/UX meant hundreds of alterations and changes to the code of the app. Furthermore, the difficulty in finding the balance between a functioning app with basic UI/UX and a professional grade app with very developed UI/UX led to many periods of distress and difficulty finding the right balance. As a team, we want to be able to put out a product that is easy to use, pleasing to the eye, and professional grade, but because of the limited time frame, as well as minimal funding, it is difficult to find the right balance between professional and functional.

\subsection{Promotion}

A challenge that is considered an ongoing challenge and one that may never be resolved is promotion or the advertisement of the mobile app and PantryGo as a whole. First off, the creation of the promotion assets, including logo design, website design, social media, flyer design, and graphic art design all require a long process of revision and perfection until satisfaction. After the theoretical creation and design of these assets are finished, there is a long process in which the small details and tedious development takes place. Additionally, many of the assets went through multiple changes and rounds of dissatisfaction, where they were completely thrown away, and created from scratch. After these assets were completed, they needed to be distributed to the target consumers and the right people who would spread the word of the application and the project even further. Difficulty in convincing people of the project and the applicability of the project was one of the hardest parts in utilizing the promotional assets and making sure it reaches the greatest number of people.

\section{SOLUTION}

PantryGo users will be able to sign up by choosing from 3 different user categories. The first category is the Foodbank profile. This profile will be used by Foodbank managers or anyone in charge of the operations at the specific food pantry. Foodbank profile users will be able to display details regarding their operational times, inventory, location, and other details that other users can view, saving time, energy, and confusion for clients and volunteers. The second category is the customer or client profile. This profile will be used by individuals or family members looking to receive food from the food pantry. Users can insert information regarding location and other personal information if desired. They will also be able to choose a grocery list with desired items, and then choose a food pantry that will automatically appear with the available items of the grocery list [13]. The third user is the volunteer profile. This user will be able to put in personal information so that other users can see the specifics of their volunteer for liability and safety reasons. The volunteers will be able to select from delivery requests by clients on that day, and will be able to see the details of the delivery, including location and pick up times. Additionally, the foodbank profiles will also be able to see customer requests that come in, and as those customers select items, the inventory within those food banks automatically change, and can be altered by the food bank manager, depending on the available inventory. Figure 1 shows the main components and flow of the app. 


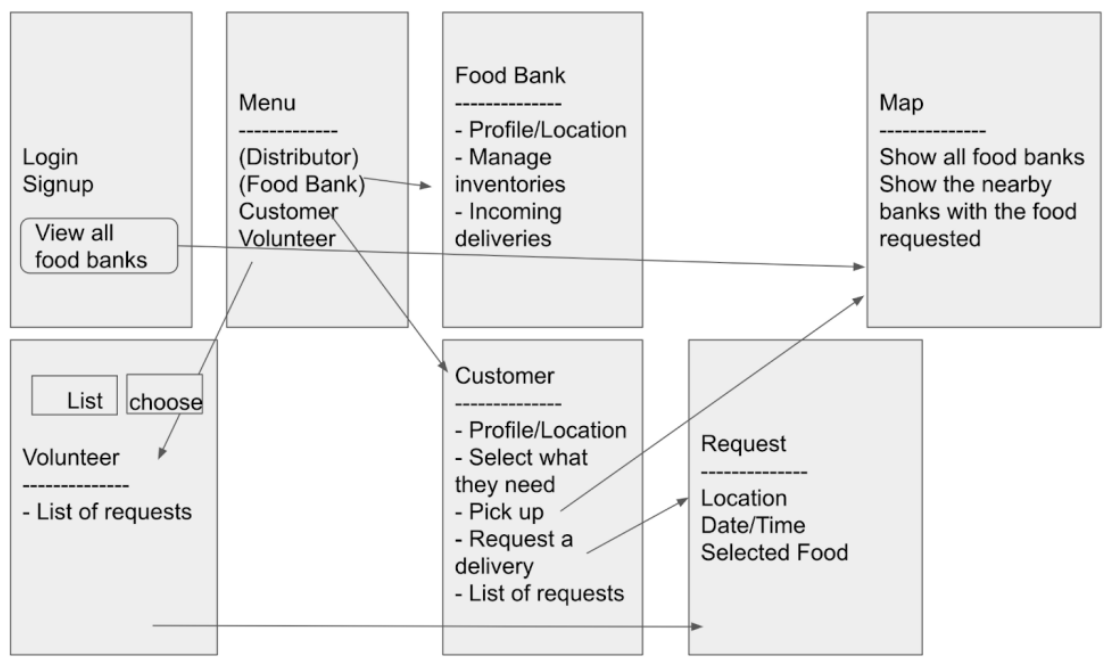

Figure 1. Overview of the app

\section{Component 1: Food Bank User}

The first component is the food bank user, in which we implemented the code to where the main functions of the food bank user is to be able to manage the inventory of the food pantry. In other words, the food bank or pantry manager will be able to change all of the food items and boxes that are available for pickup or delivery on a daily basis. Food bank users will also be able to change their locations and upload their information for display to other users such as customers and volunteers. They will also be able to see all of the food requests from customers, as well as all of the details of that request, including whether the request was completed by the volunteer or not. Figure 2 shows a segment of the code for the food bank users ability to change inventory. Figure 3 shows screenshots of the app from the food bank users perspective.

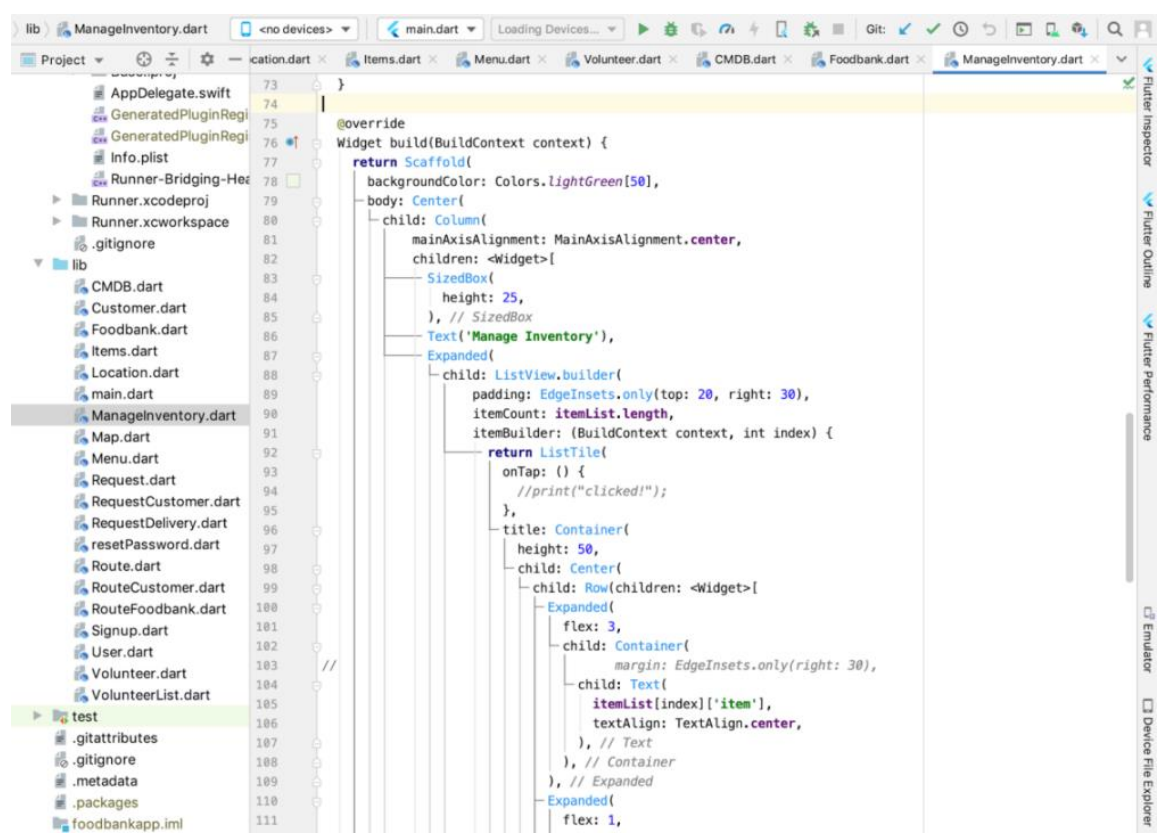

Figure 2. Food bank users ability to change inventory 


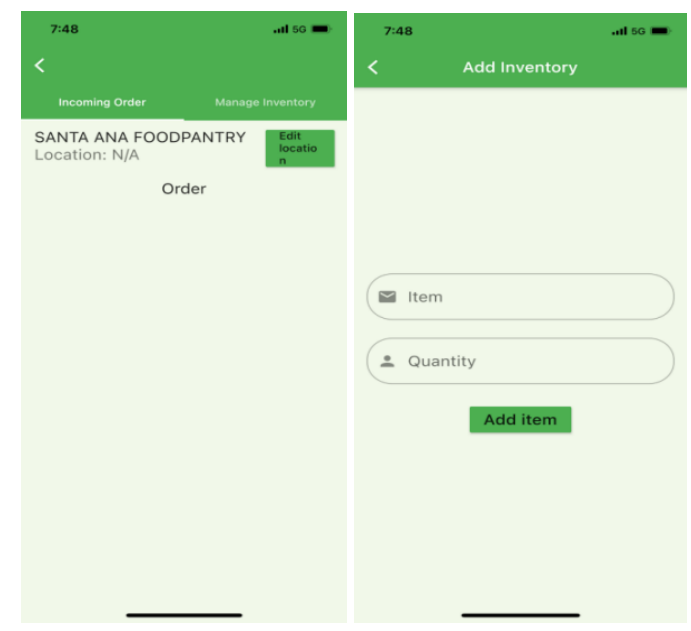

Figure 3. Food bank users perspective

\section{Component 2: Customer User}

The second component is the customer user, which has a few main functions. The customer will be able to request a food list based on the available items from a food pantry within their area, and through artificial intelligence, the food items which they selected will automatically link with a food pantry with the specified items. Customers will also be able to see all of their past requests and the details of the request, and when a volunteer selects that customers request, it will automatically move into request history as completed. Figure 4 shows a segment of the code that allows customers to request specific food items, while Figure 5 shows screenshots from the viewpoint of the customer user.

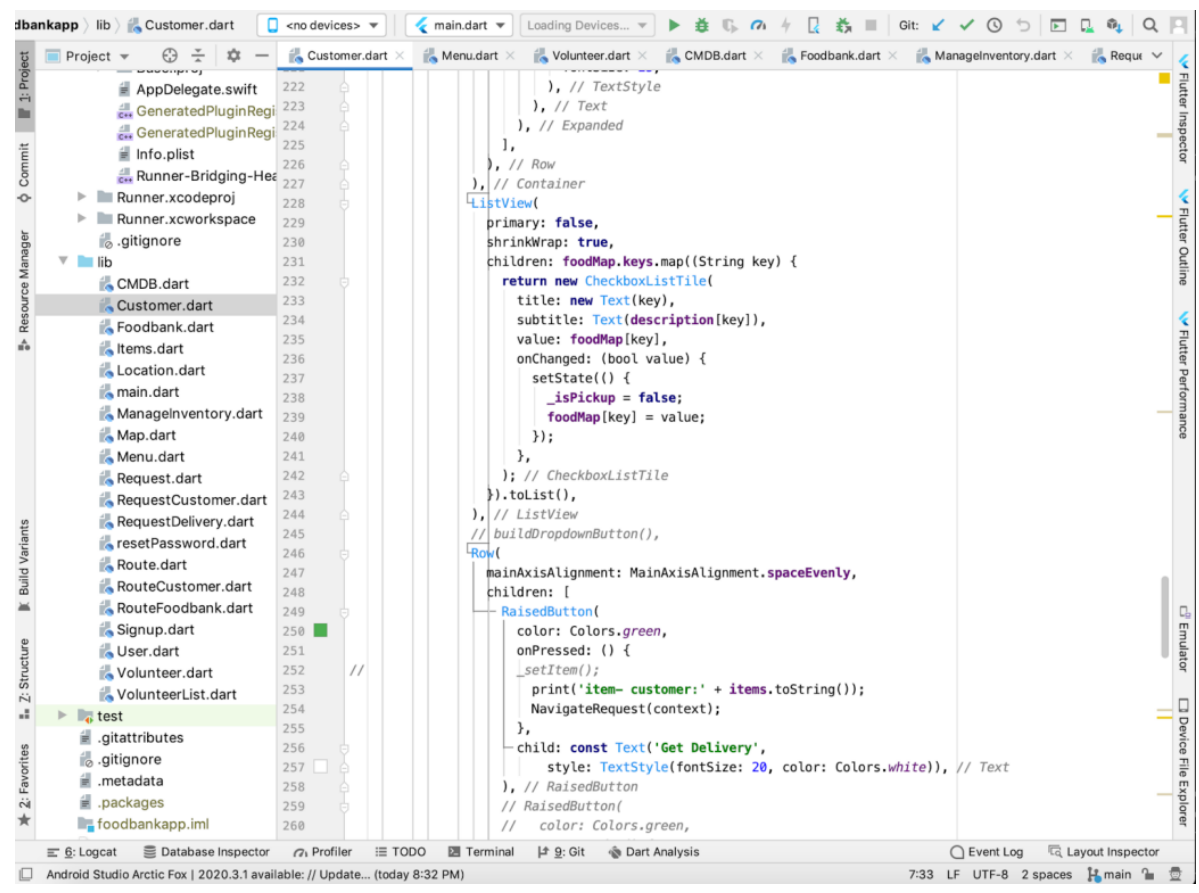

Figure 4. Customers request specific food items 


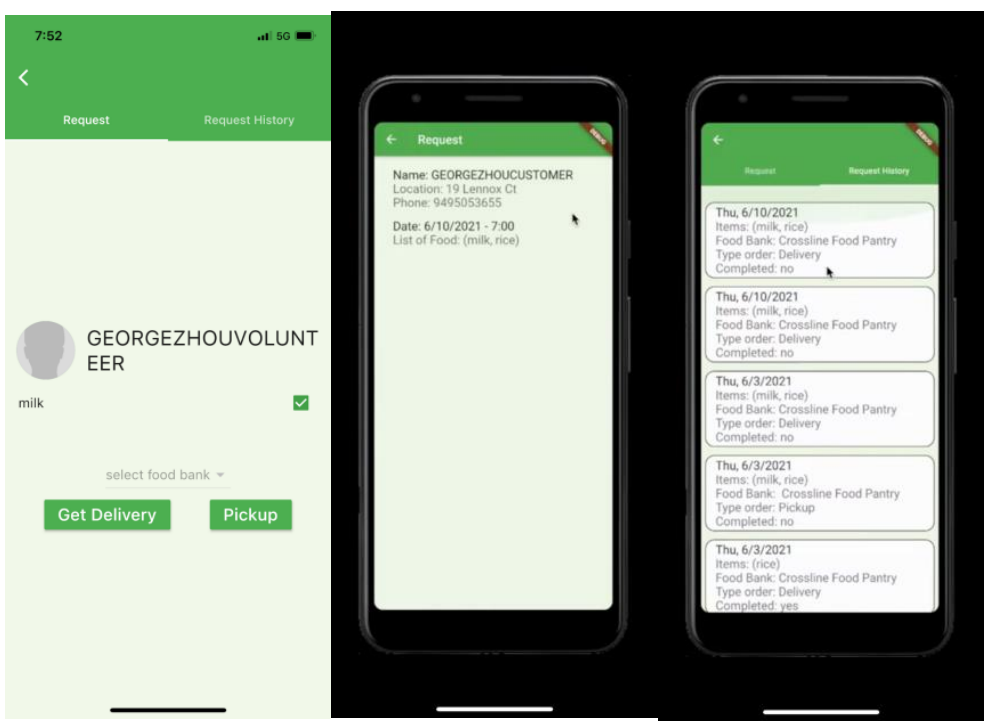

Figure 5. Screenshots from the viewpoint of the customer user

Component 3: Volunteer User

The third component is the volunteer user, which has the main functions of selecting customer food requests. The volunteer will automatically receive all food requests made by any customer at any time, including all the details retained to the request, such as location, time, date, and food items. Additionally, once the volunteer selects the request, it will go to the list of customers, and once completed, will update the screens on the other two users. In Figure 6, it will show the code that allows volunteers to select customer requests, and in Figure 7, it shows screenshots from the view of the volunteer user.

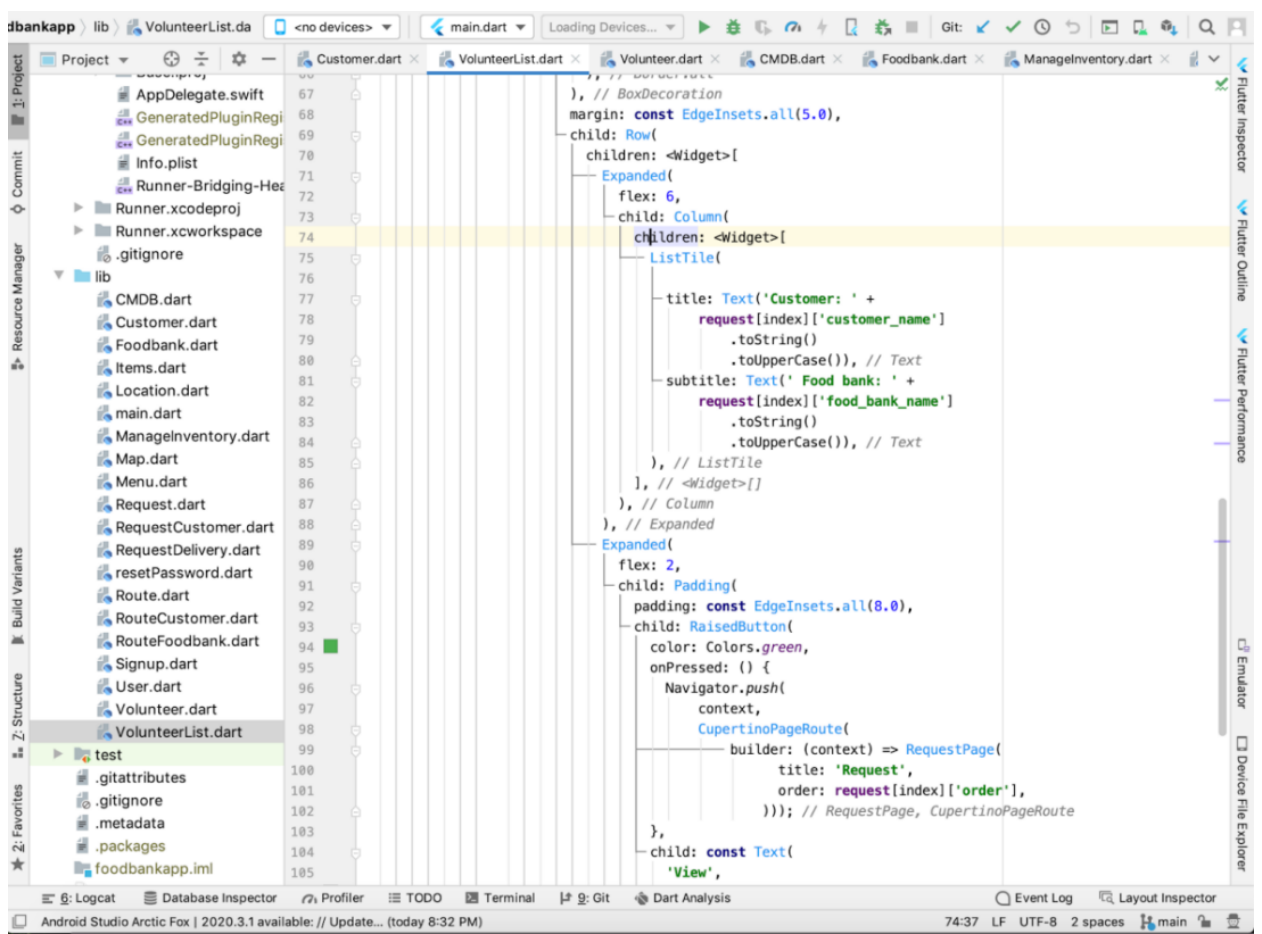

Figure 6. Volunteers select customer requests 


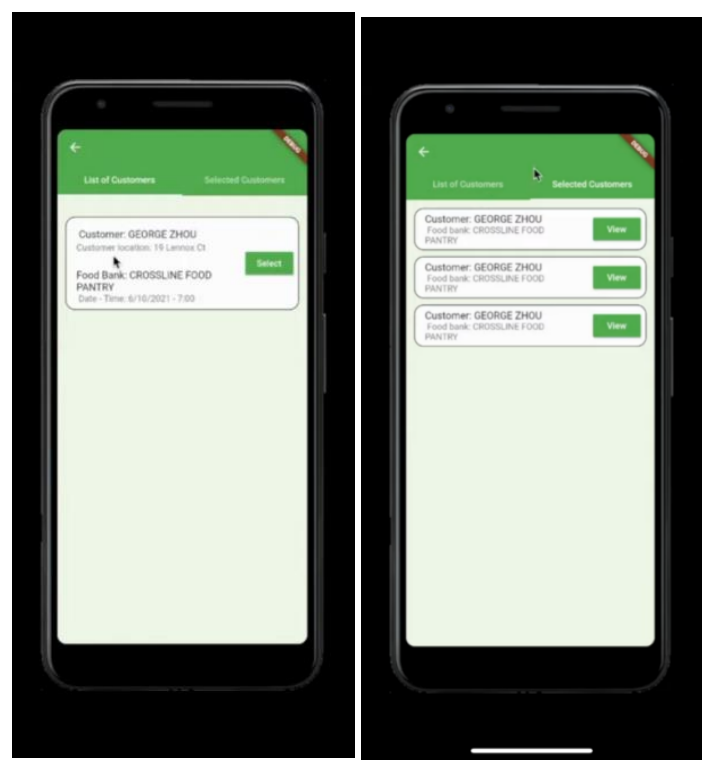

Figure 7. Screenshots from the view of the volunteer user

The connection between the 3 components comes in the form of steps. The first step involves the food bank user, who will insert the inventory and the quantity of each specific item. Next, the customer user will be able to see the food items from the selective food pantry, and will then select the items they desire, and select the date and time on which they would like the request delivered [15]. Then, that request will be sent to both the food pantry and the volunteer, where the volunteer will select that food request and pick up the food from the food pantry, and then deliver it to the home of the individual or family.

\section{EXPERIMENT}

To be able to evaluate the efficiency of our approach, we gathered data and information over a 2 week span from the Crossline Church food pantry through the tallying of requests, weighing of food waste, and surveying of customers and volunteers. Two experiments were conducted to test the solution and its effectiveness. The first experiment explores the effects of the application on food waste, and the second experiment evaluates the applications effect on volunteer involvement.

\section{Experiment 1: PantryGo's effect on Food Waste}

This experiment involves the collection of data of the amount of food waste left over after each week. The first week was monitored without the usage of our solution, and the second week is monitored with the implementation of the application. Food waste has become a global crisis, especially in the United States, where surpluses of food has led to detrimental effects to the environment as a carbon footprint. The application attracts more customers to the food pantry and expands its reach, effectively reducing the food waste that piles up at locations such as food pantries. In Figure 8, it shows the pounds of food waste left over after week 1 and week 2. Clearly, the amount of food waste was reduced by more than $50 \%$ after the implementation of the application, showing its effectiveness in greater food pantry usage. 


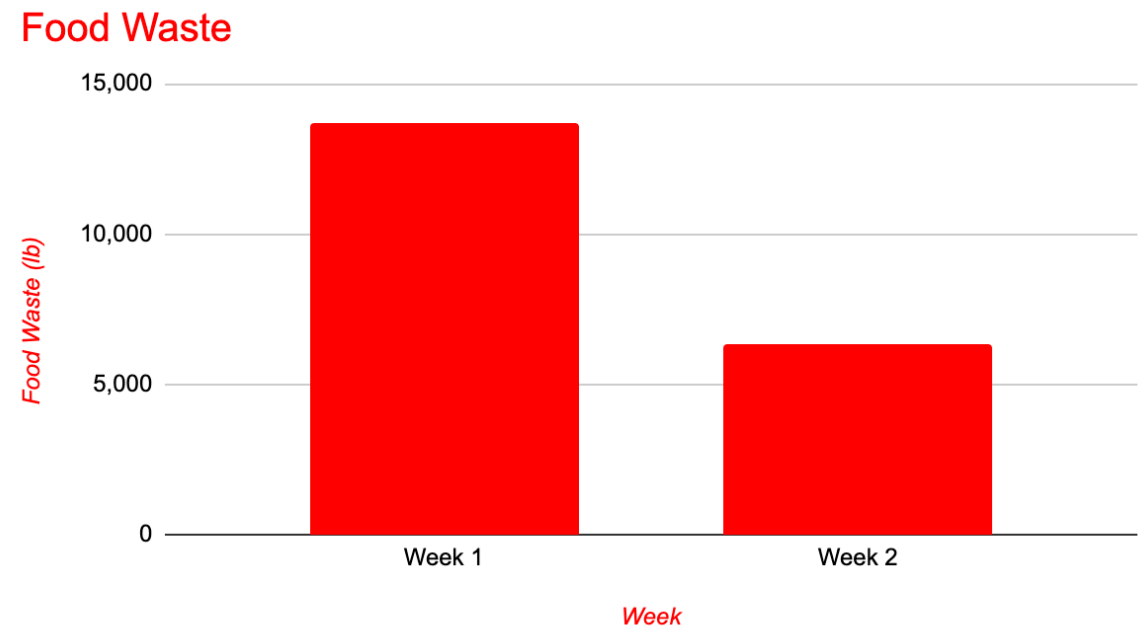

Figure 8. Food waste

\section{Experiment 2: PantryGo's effect on Volunteer Involvement}

This experiment involves the gathering of data and information based on surveys given to different youth volunteer groups asking for whether they were interested in volunteering for the food pantry. The first survey (during the first week) was given before the control group was notified of the opportunity to serve as delivery drivers, while the second survey (during the second week) was given right after the group was notified of the existence of the app. Figure 9, week 1 , shows the number of volunteers who committed to volunteer for the food pantry that week in multiple positions except for food delivery driver, while week 2 shows the number of volunteers committed to work in multiple positions including food delivery driver. The figure evidently shows that there was an increase in volunteers in the second week, having linear correlation to the introduction of the application or solution. Therefore, teenage volunteers will have more involvement and interest in volunteering when given the opportunity to be a delivery driver, as these teenagers tend to enjoy car rides over physical labor.

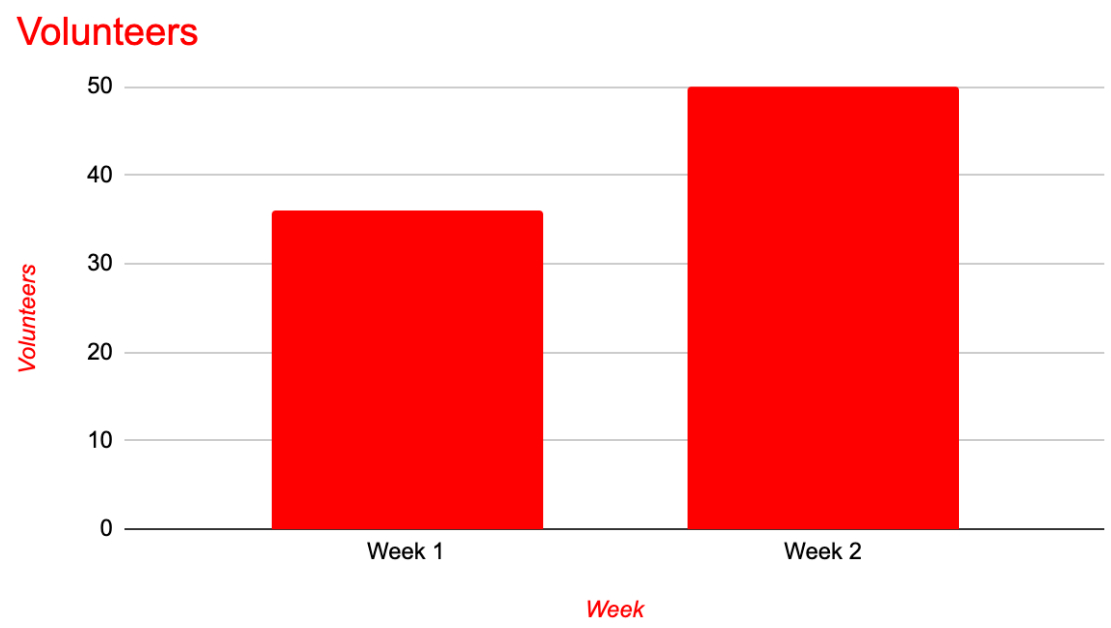

Figure 9. Volunteers 
Both experiments show the effect on which the application or solution has addressed the problems faced within food banks and pantries, ranging from excess food waste or limited volunteers. The experiments showed that both of theses aspects were benefited after the introduction and implementation of PantryGo, both decreasing food waste and increasing volunteer involvement. These results reach my expectation in the positive benefits the solution brings to the food pantry system and current condition.

\section{RELATED WORK}

Leah R. Kicinski in her publication "Characteristics of Short and Long-Term Food Pantry Users"[1] discusses the modern research findings of the eating habits and overall health of frequent food pantry visitors. Kickiski and her team of researchers at Grand State Valley University used an interview based data collections strategy within food insecure residents in the Michigan area. Her findings indicate that food pantries are catering to two distinct group of people, one consisting of recently unemployed individuals, or families who have been under the poverty line for over a decade. Similar to my work, this paper discusses the flaws within the food pantry system, including its lack of accessibility, but does not give a solution to these problems, as Kicinski states, "Pantry users are not all identical in their characteristic, reasons for food need, or their food pantry experiences" [2]. Kicinski's generalized statement only mentions the culprit behind the reason for the food pantries' lack of accessibility, while my paper discusses a solution that can cater to all of the differences that cause different constraints.

"The Cost of Free Assistance: Why Low Income Individuals Do Not Access Food Pantries", published by Kelley Fong, Rachel A. Wright, and Christopher Wimer discusses the possible reasons why many food insecure families do not utilize the free resources provided by food pantries. For most of these families, constraints including minimal transportation, distance, working multiple jobs, illnesses, or fast food options often lead them to ignore the resources provided by food pantries. This publication released by the Department of Sociology at Harvard University goes into great detail regarding certain research findings and data regarding the status of these low income families, and similar to my paper, give possible solutions for food pantries to meet these families in the middle.

In a very recently published paper titled "A Community Partnership for Home Delivery of Food Boxes to Covid-19 Quarantined and Isolated Families" led by a number of authors headed by Emily English examines the partnership between a community group that delivers food boxes to families who are isolated due to illnesses or COVID-19 cases, barring them from shopping for groceries. The community group, already connected to this community of residents, were able to deliver to over 531 families within the area. The publication, in its summary of the project's accomplishments and mission, is very similar to some of the main motives of PantryGo and its target market. The article also goes into great detail regarding the specific transfer strategies utilized, as they state, "Addresses were then sent to the regional transit partner to create routes and secure the appropriate number of buses and drivers to be utilized for deliveries" [3]. Similar to my publication, the addresses of these families were mentioned in ensuring the process of delivery is efficient and safe.

\section{Conclusions}

Because of the increasing demand for food pantry resources and distribution due to the ongoing pandemic, causing unemployment rates to skyrocket, I have proposed an application that can be used to expand the reach and accessibility of food pantries. The application uses artificial intelligence to fluidly offer users to choose their desired food items, linking these families or 
individuals with food pantries and volunteers, who will deliver their specific food requests directly to their door. This allows for families who experience constraints, barring them from reaching their local food pantry, including illnesses, limited transportation, large distances, multiple jobs, or time constraints to be able to still access the free resources provided by their local food pantry. To evaluate the effectiveness of this application, two separate experiments were conducted using gathered information through the form of food waste and volunteer involvement. The first experiment measured the change in food waste left over after a week of food pantry operation with and without the implementation of the application. The amount of food waste decreased drastically after the use of the application, showing its immediate effect in increasing the usage of the food pantry and its resources. Furthermore, the application can also benefit restaurants and grocery stores in reducing its food waste and carbon footprint by bringing its surplus of food to food pantries, and then to families who are experiencing food insecurity. The second experiment consisted of taking surveys of volunteers before and after the introduction of the application. The results showed that volunteers were much more likely to commit to volunteering when the opportunity of becoming a delivery driver was offered, furthermore showing the applications ability to attract teenage volunteers and community collaboration. The overall results of these two experiments validate the applicability of the solution, as well as its estimated success and efficiency.

The current limitations of the application is expanding the project to other food pantries and banks in different regions or states. Currently, the application is running in Southern Orange County, where the food pantry serves many residents in Orange County. PantryGo hopes to spread to other states and regions where the need to this application is at an even greater level, but because of its relatively early stages in operation, it is difficult to sell the idea to other food pantry managers or systems. These food pantries often already have a system of distributing their food to their community, and have worked hard to make that system as efficient as possible.

PantryGo hopes to find other collaborators or leaders within those different states or regions to represent the application and promote it similar to that of which is occurring in Orange County. These representatives in each region can be regional managers or board members of the organization, and can collaborate with other board members or regional leaders to grow as a family within PantryGo and spread the application nationwide.

\section{REFERENCES}

[1] Kicinski, Leah R. "CHARACTERISTICS OF SHORT AND LONG-TERM FOOD PANTRY USERS. " Michigan Sociological Review, vol. 26, 2012, pp. 58 - $74 . \quad$ JSTOR, www.jstor.org/stable/23292651. Accessed 5 Aug. 2021.

[2] Fong, K.; Wright, R.; Wimer, C. The cost of free assistance: Why low-income individuals do not access food pantries. J. Sociol. Soc. Welf. 2016, 43, $71-93$.

[3] Emily English, Christopher R. Long, Krista Langston, Bonnie Faitak, April L. Brown, Amanda Echegoyen, Joel Gardner, Casey Cowan, Debbie Rambo, Brenda Perritt, Barb Laubenstein, Alyssa Snyder, Pat Bourke, Melisa Lelan\& Pearl A. McElfish (2021) A Community Partnership for Home Delivery of Food Boxes to COVID-19 Quarantined and Isolated Families, Journal of Hunger \& Environmental Nutrition, 16:1, 19-28, DOI: 10.1080/19320248.2020.1863284

[4] Gundersen, Craig, and James P. Ziliak. "Food insecurity and health outcomes." Health affairs 34.11 (2015): 1830-1839.

[5] Bhattarai, Gandhi Raj, Patricia A. Duffy, and Jennie Raymond. "Use of food pantries and food stamps in low - income households in the United States." Journal of Consumer Affairs 39.2 (2005): 276-298.

[6] Layard, Richard, Stephen Nickell, and Richard Jackman. "The unemployment crisis." (1994).

[7] He, Feng, Yu Deng, and Weina Li. "Coronavirus disease 2019: What we know?." Journal of medical virology 92.7 (2020): 719-725. 
[8] Arney, Fiona, and Dorothy Scott, eds. Working with vulnerable families: A partnership approach. Cambridge University Press, 2013.

[9] De Reuver, Mark, Carsten Sørensen, and Rahul C. Basole. "The digital platform: a research agenda." Journal of Information Technology 33.2 (2018): 124-135.

[10] Gale, David. "The law of supply and demand." Mathematica scandinavica (1955): 155-169.

[11] Burkhart, Patrick J., and Suzanne Reuss. Successful strategic planning: A guide for nonprofit agencies and organizations. Sage, 1993.

[12] Gordon, Jeffry S., and Ryan McNew. "Developing the online survey." Nursing Clinics of North America 43.4 (2008): 605-619.

[13] Bassett, Raewyn, Brenda Beagan, and Gwen E. Chapman. "Grocery lists: connecting family, household and grocery store." British Food Journal (2008).

[14] Holmes, Eleanor, et al. " "Nothing is going to change three months from now" : A mixed methods characterization of food bank use in Greater Vancouver." Social Science \& Medicine 200 (2018): 129-136.

[15] Greenberg, Michael, Gwendolyn Greenberg, and Lauren Mazza. "Food pantries, poverty, and social justice." (2010): 2021-2022.

(C) 2021 By AIRCC Publishing Corporation. This article is published under the Creative Commons Attribution (CC BY) license. 